\title{
Video Article \\ Continuous Blood Sampling in Small Animal Positron Emission Tomography/ Computed Tomography Enables the Measurement of the Arterial Input Function
}

\author{
Teresa Mann ${ }^{1}$, Jens Kurth ${ }^{2}$, Anne Möller ${ }^{3}$, Joanna Förster ${ }^{3}$, Brigitte Vollmar ${ }^{4}$, Bernd J. Krause ${ }^{2}$, Andreas Wree ${ }^{1}$, Jan Stenzel ${ }^{3}$, Tobias Lindner ${ }^{3}$ \\ ${ }^{1}$ Institute of Anatomy, Rostock University Medical Center \\ ${ }^{2}$ Department of Nuclear Medicine, Rostock University Medical Center \\ ${ }^{3}$ Core Facility Multimodal Small Animal Imaging, Rostock University Medical Center \\ ${ }^{4}$ Rudolf-Zenker-Institute for Experimental Surgery, Rostock University Medical Center \\ *These authors contributed equally
}

Correspondence to: Teresa Mann at Teresa.Mann@med.uni-rostock.de

URL: https://www.jove.com/video/59701

DOI: doi:10.3791/59701

Keywords: Medicine, Issue 150, PET imaging, kinetic modeling, quantification, radiotracer uptake, continuous blood sampling, arterial cannulation, arteriovenous shunt, arterial input function, small animal, rodent, rat

Date Published: 8/8/2019

Citation: Mann, T., Kurth, J., Möller, A., Förster, J., Vollmar, B., Krause, B.J., Wree, A., Stenzel, J., Lindner, T. Continuous Blood Sampling in Small Animal Positron Emission Tomography/Computed Tomography Enables the Measurement of the Arterial Input Function. J. Vis. Exp. (150), e59701, doi:10.3791/59701 (2019).

\section{Abstract}

For quantitative analysis and bio-kinetic modeling of positron emission tomography/computed tomography (PET/CT) data, the determination of the temporal blood time-activity concentration also known as arterial input function (AIF) is a key point, especially for the characterization of animal disease models and the introduction of newly developed radiotracers. The knowledge of radiotracer availability in the blood helps to interpret PET/CT-derived data of tissue activity. For this purpose, online blood sampling during the PET/CT imaging is advisable to measure the AIF. In contrast to manual blood sampling and image-derived approaches, continuous online blood sampling has several advantages. Besides the minimized blood loss, there is an improved resolution and a superior accuracy for the blood activity measurement. However, the major drawback of online blood sampling is the costly and time-consuming preparation to catheterize the femoral vessels of the animal. Here, we describe an easy and complete workflow for catheterization and continuous blood sampling during small animal PET/CT imaging and compared it to manual blood sampling and an image-derived approach. Using this highly-standardized workflow, the determination of the fluorodeoxyglucose $\left(\left[{ }^{18} \mathrm{~F}\right] \mathrm{FDG}\right)$ AIF is demonstrated. Further, this procedure can be applied to any radiotracer in combination with different animal models to create fundamental knowledge of tracer kinetic and model characteristics. This allows a more precise evaluation of the behavior of pharmaceuticals, both for diagnostic and therapeutic approaches in the preclinical research of oncological, neurodegenerative and myocardial diseases.

\section{Video Link}

The video component of this article can be found at https://www.jove.com/video/59701/

\section{Introduction}

Positron emission tomography/computed tomography (PET/CT) is a nuclear imaging technology that enables visualization of metabolic processes in the body following injection of a radioactively labeled ligand, also called tracer. Whereas the ligand is a molecule that is involved in a metabolic pathway or targets cell surface proteins, the radioactive label is a positron-emitting radionuclide. Gamma rays are indirectly emitted by the positron decay and allow the detection of its distribution in the organism with extracorporeal PET detectors. In this way, different cellular molecules can be targeted: neurotransmitter receptors and transporters, metabolic processes like glycolysis or mitochondrial proteins like the translocator protein $18 \mathrm{kDa}$ (TSPO) to detect activated glia cells.

In preclinical research, PET/CT is an attractive method to study biochemical processes in a non-invasive manner in vivo, thus allowing longitudinal studies. PET/CT data support the analyses of disease mechanisms, the assessment of the characteristics and pharmacokinetics of new drugs and the validation of both, current and novel radiotracers for translational research.

During PET/CT analyses three tracer states can be defined (example of the 2-tissue compartment model): First, the tracer flows within the blood after its application (state 1; conc. [blood]). Second, it enters the tissue via the capillary bed and can there either freely move within the extracellular space or is unspecifically bound to diverse cellular or extracellular structures (state 2; conc. [unspec]). Third, the tracer can be specifically bound (with or without metabolic trapping) to its target molecule (state 3, conc. ${ }_{\text {[spec] }}$ ). All these dynamic processes between the compartments are to some extent bidirectional and the diffusion processes are described by rate constants (K1, $\mathrm{k} 2, \mathrm{k} 3$, and $\mathrm{k} 4)$. While the concentration of the tracer in the blood (i.e., state 1) is called "Input", the concentration of unspecifically and specifically bound tracer (i.e., state 2 and state 3 ) is 
called "Output" and can be directly derived from the PET Image. This physiological relation can be displayed in the 2-tissue compartment model (Figure 1).

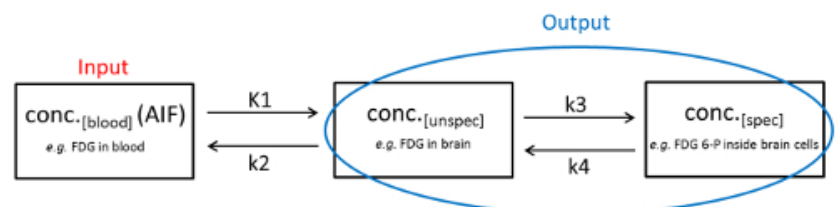

Figure 1: The two-tissue compartmental model. The physiological conditions of the three different tracer states and the dynamic processes between them are displayed. Please click here to view a larger version of this figure.

In the ideal case, conc. ${ }_{\text {[spec] }}$ is proportional to the concentration of its target molecule. However, the output of the PET/CT measurement is the

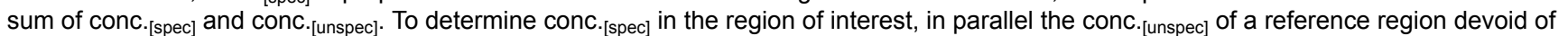
the target protein/pathway is determined. By using appropriate mathematical equations one can now calculate conc. [spec], most commonly using the compartment model (a bio-kinetic modeling approach). However, in many cases, such a reference region devoid of the target protein is not available $^{1,2}$. In these cases, the conc. ${ }_{\text {[blood] }}$ can be used to determine conc. ${ }_{\text {[spec] }}$. Since the conc. .blood] $_{3}$ is varying due to different liver and kidney clearance, excretion, blood flow, different brain-blood barrier penetration and disease-related factors ${ }^{3}$, the current gold standard is to measure the conc. [blood] in parallel to the PET/CT scan by continuous blood sampling. This gives the arterial input function (AIF), which is defined as conc. [blood] over time ${ }^{4}$. Of note, performing continuous blood sampling is considered technically highly challenging, especially in small animals such as rats or mice ${ }^{5}$.

Here, we provide an easy and practical protocol to continuously sample blood from rats via an arteriovenous (a-v) shunt between the femoral vein and artery. Coupled to a commercially available detector-pump system, we are able to generate a real-time, continuous AIF during dynamic $\left[{ }^{18} \mathrm{~F}\right]$ fluorodeoxyglucose $\left(\left[{ }^{18} \mathrm{~F}\right] \mathrm{FDG}\right)-\mathrm{PET} / \mathrm{CT}$ scans in rats and compared it to alternative approaches. PET/CT imaging was performed in male sprague dawley rats at an age of 4 months with an average weight of $462 \mathrm{~g} \pm 33 \mathrm{~g}$ (mean \pm standard deviation) using a multimodality PET/CT scanner.

Since a wide variety of devices is used during the series of measurements (dose calibrator, online blood sampler, PET/CT, and well counter), a quality control procedure referred as cross calibration is needed to check the quantitative accuracy of all systems and to compensate for differences. Cross calibration in the context of online blood sampling means that the count rate for a given activity concentration measured in corrected PET images can be converted into the concentration measured with the twilite system for the same concentration. Therefore, a cross calibration procedure between PET/CT, blood sampling system, and well counter has been established.

This highly standardized methodology provides a powerful approach to quantify metabolic and cellular processes in preclinical small animal research and is an elegant way to improve the reliability and reproducibility of the AIF. The AIF can then be used to quantify the specifically bound tracer in tissue in preclinical PET/CT data using bio-kinetic modeling.

\section{Protocol}

All animal handling and experiments were approved by the state Animal Research Committee of Mecklenburg-Western Pomerania (LALLF MV/7221.3-1.1-004/18, approval: 03.04.2018). The experiments were performed in compliance with the ARRIVE Guidelines.

NOTE: Animals were kept under standard conditions $\left(22 \pm 2{ }^{\circ} \mathrm{C}, 12 \mathrm{~h}\right.$ day-and-night cycle) with water and food ad libitum. All needed equipment for the preparation of the shunt system, the operation procedure and the actual measurements are listed in the Table of Materials.

\section{Preparation and surgical procedure for catheterization of the animal}

1. Fast the animal for at least $12 \mathrm{~h}$ with free access to water. For anesthesia, place the rat in an induction chamber and fill it continuously with oxygen/isoflurane mix. For initiation use $2.5-3.5 \%$ isoflurane and for maintenance $1.5-3.0 \%$ (flow rate of $1.2-1.5 \mathrm{~L} / \mathrm{min}$ ). NOTE: Fasting is necessary for studies using the tracer $\left[{ }^{18} \mathrm{~F}\right] \mathrm{FDG}$ but not for other tracers. Measuring glucose blood levels using manual blood draws described in section 4 is recommended to ensure stable values or to correct for in kinetic modeling.

2. Place the anesthetized rat in dorsal position on a heating mat, under the surgical microscope and add vet ointment on its eyes. Monitor and maintain the body temperature of the rat continuously during the experiment $\left(37 \pm 0.5^{\circ} \mathrm{C}\right)$ with a rectal probe.

3. Tape the legs of the rat to the work surface to hold the legs in position. Disinfect the operating site with a mucosal disinfectant and shave the leg and crotch (operation side) of the rat. Finish with a final cleansing with the disinfectant.

4. Make an incision of about $20 \mathrm{~mm}$ using surgical forceps and scissors at the groin of the rat. Dissect the fine skin layers and expose the femoral vein, artery and nerve with the micro forceps. Place two fine filaments under each femoral vein and artery.

5. Seal vein and artery with each distal filament and hold under tension with a bulldog clamp. Use the proximal suture filaments to tension the vessel using the bulldog clamps (without a knot).

6. Block the vein with an aneurysm clamp proximal, but 2-3 $\mathrm{mm}$ distal from the suture with the bulldog clamp. Use corneal scissors to make a small incision into the vein ( $1 / 3$ of the diameter) and remove leaking blood with a sterile cotton swap. Dilate the vein with a dull forceps and hold it open. Insert the sharpened catheter (inner diameter [ID]: $0.58 \mathrm{~mm}$, outer diameter [OD]: $0.96 \mathrm{~mm}$ ) into the vein and push it in proximal direction, up to the aneurysm clip.

7. Open the aneurysm clip and push the catheter further in proximal direction (approximately $2-3 \mathrm{~cm}$ ), if the catheter is placed right, blood will flow into the catheter. Secure the catheter with the proximal suture by making two knots; if necessary, place an additional suture around the vein and the catheter. Check the functionality of the catheter by flushing and aspirating with an insulin syringe (30 G needle) filled with $100 \mu \mathrm{L}$ of heparinized saline solution (50 units $/ \mathrm{mL})$. 
8. Place the catheter in the artery by repeating steps 1.6 and 1.7 .

9. When both catheters are correctly placed, close the leg with sutures and carry the animal to the PET/CT.

NOTE: Be as careful as possible with the catheters during transport of the animal, otherwise shifting of the catheter might occur.

\section{Setup of the shunt system}

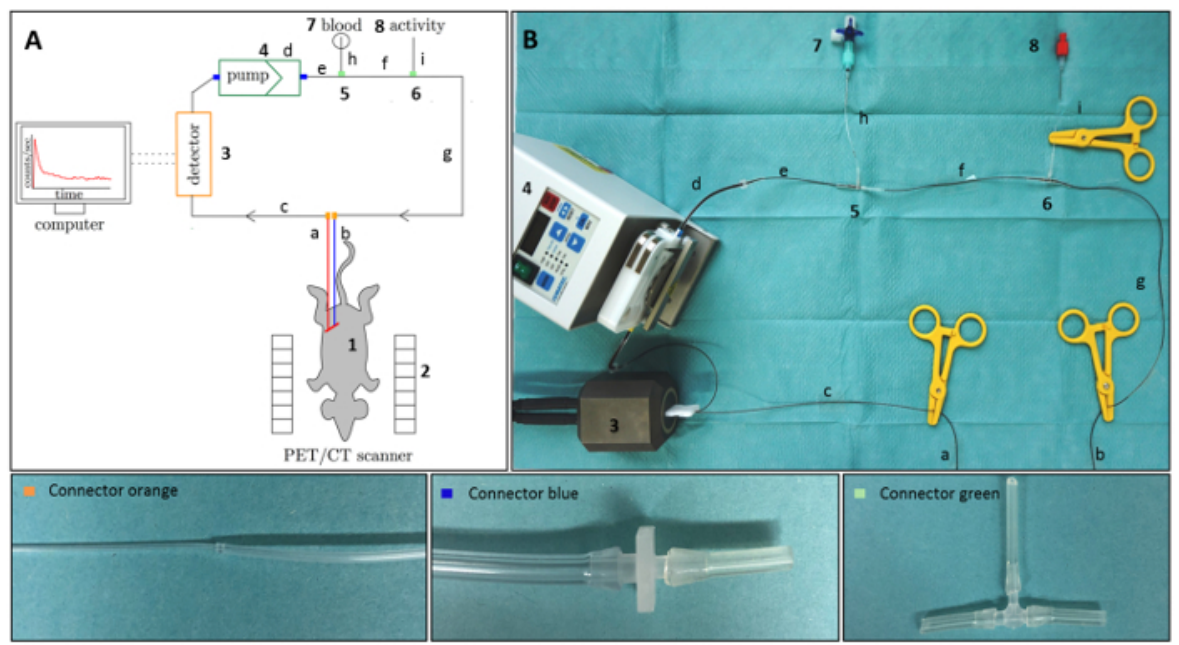

Figure 2: Scheme of the measurement setup. (A) Schematic drawing of the measurement setup. (B) Photo of the connected shunt system with the twilite detector, peristaltic pump and different connector types. The time-course of radioactivity in blood of a rat is detected while the animal (1) is scanned in the PET/CT (2). Therefore the arterial (a) and venous (b) catheter is connected to the detector pump system via adapter pieces (connector orange, connector blue and connector green). The arterial blood is then pumped from the arterial catheter through the detector (3) to a peristaltic pump (4) and back into the body via the venous catheter. A 3-way valve (7) is integrated in the tube system to perform tracer injection, manual blood draws and rinsing. A T-piece (8) is assembled to inject activity. The detector is connected with a computer to view, calibrate and correct the continuous blood data. Please click here to view a larger version of this figure.

1. Cut off 6 parts of the fine bore polythene tubing (FBPT) (ID: $0.58 \mathrm{~mm}, \mathrm{OD}: 0.96 \mathrm{~mm}$ ) with a length of $\mathbf{c}=735 \mathrm{~mm} ; \mathbf{e}=100 \mathrm{~mm} ; \mathbf{f}=171 \mathrm{~mm}$, $\mathbf{g}=875 \mathrm{~mm} ; \mathbf{h}=90 \mathrm{~mm}$ and $\mathbf{i}=75 \mathrm{~mm}$ (Figure 2). Cut off 8 parts of the silicone pump tubes (black/black/black, ID: $0.76 \mathrm{~mm}, \mathrm{OD}: 2.48 \mathrm{~mm}$ ) with a length of approximately $20 \mathrm{~mm}$.

2. Place reduction connectors (from ID $2.5 \mathrm{~mm}$ to ID $1.5 \mathrm{~mm}$ ) on both ends of the silicone pump tube d (yellow/blue/yellow, ID: $1.52 \mathrm{~mm}$, OD: $3.20 \mathrm{~mm}$ ). Put a prepared $20 \mathrm{~mm}$ part of the silicone tubes (black/black/black) on the other end of the used reduction connectors (see connector blue in Figure 2).

3. Place the prepared part $\mathbf{c}$ of the FBPT in the assembled connector blue on the one end of the silicone pump tube $\mathbf{d}$ (yellow/blue/yellow) and the prepared part e of the FBPT in the assembled connector blue on the other end. Put a prepared $20 \mathrm{~mm}$ part of the silicone tubes (black/ black/black) on the ends of two T-pieces 5 and 6 (tube T-connector ID: $1.5 \mathrm{~mm}$; see connector green in Figure 2).

4. Connect the free end of part $\mathbf{e}$ of the FBPT to the left side of the assembled connector green (5) and place the prepared part $\mathbf{f}$ of the FBPT at the opposite side of connector green (5). Place the free end of part $f$ of the FBPT in left side of assembled connector green (6) and place the prepared part $\mathbf{g}$ of the FBPT at the opposite side of connector green (6). Add the prepared part $\mathbf{h}$ of the FBPT to the free end of the assembled connector green (5) and the prepared part $\mathbf{i}$ of the FBPT to the free end of the assembled connector green (6).

5. Connect a combi-stopper to a hypodermic needle ( $\mathrm{G} 23 \times 11 / 4 " / \varnothing 0.60 \mathrm{~mm} \times 30 \mathrm{~mm})$ and add it to a three-way valve. Place the prepared three-way valve with the needle in the free end of the part $\mathbf{h}$ of the FBPT. Connect a combi-stopper to a hypodermic needle and place the needle in the free end of the part $\mathbf{i}$ of the FBPT.

NOTE: Before starting the online blood sampling, see section 5.

6. Put the free ends of part $\mathbf{c}$ and $\mathbf{g}$ of the FBPT into a $100 \mathrm{~mL}$ beaker filled with $20 \mathrm{~mL}$ of heparinized saline solution ( $50 \mathrm{units} / \mathrm{mL}$ ). Start the peristaltic pump with a flow rate of $1.52 \mathrm{~mL} / \mathrm{min}$ so that the shunt system is completely filled with the physiological saline solution. Afterwards set three scissor clamps at the ends of part $\mathbf{c}$ and $\mathbf{g}$ and in the middle of part $\mathbf{i}$ of the FBPT.

7. Release the scissor clamps from part $\mathbf{c}$ and $\mathbf{g}$ of the FBPT. Connect the arterial catheter $\mathbf{a}$ to the free end of part $\mathbf{c}$ of the FBPT and connect the venous catheter $\mathbf{b}$ to the free end of part $\mathbf{g}$ of the FBPT (see connector orange in Figure 2 ).

\section{Image acquisition and reconstruction}

1. Place the animal in head-prone position on the shuttle bed pallet $(70 \mathrm{~mm})$. Control respiration of the rat and keep body temperature at $37 \pm$ $0.5^{\circ} \mathrm{C}$ using a heating pad and a rectal probe throughout image acquisition. Move the shuttle bed to the extended bed position for injection (pre-acquisition) and connect the inserted catheters to the shunt system.

2. Keep the animal under anesthesia with isoflurane $(2.5 \%$ isoflurane in oxygen, flow rate $1.2-1.5 \mathrm{~L} / \mathrm{min})$ via a nose cone.

3. Start the peristaltic pump with a flow rate of $1.52 \mathrm{~mL} / \mathrm{min}$ to fill the shunt system with the blood of the animal. Move the shuttle bed to the center of the field of view of the PET detection ring and start the online blood sampling system (see section 5 ).

4. Start the PET/CT workflow using parameters described in section 3.5 after $60 \mathrm{~s}$ and subsequently inject a dose of approximately $22 \mathrm{MBq}$ $\left[{ }^{18} \mathrm{~F}\right] \mathrm{FDG}$ in a volume of about $0.5 \pm 0.1 \mathrm{~mL}$ intravenously via the T-piece. Flush the T-piece with about $150 \mu \mathrm{L}$ of heparinized saline solution afterwards.

5. Acquire a dynamic PET over 60 min and a CT scan at the end of the PET imaging. 
1. For PET emission acquisition, set $3600 \mathrm{~s}(60 \mathrm{~min})$ in the acquire by time option. Select $\mathrm{F}-18$ as study isotope and use $350-650 \mathrm{keV}$ as energy level and 3,438 ns as timing window.

2. For CT acquisition, select attenuation scan in the acquisition option. In the projection settings field, choose 120 projection for a half total rotation. For field of view (FOV) and resolution settings, select low as magnification and $4 \times 4$ as binding with $275 \mathrm{~mm}$ axial scanning length and $3328 \mathrm{px}$ as transaxial CCD size. In the exposure settings field, set $500 \mu \mathrm{A}$ for current, $80 \mathrm{kV}$ for voltage and $180 \mathrm{~ms}$ for exposure time.

3. For PET emission histogram, set a series of 20 frames $(6 \times 10 \mathrm{~s}, 8 \times 30 \mathrm{~s}, 5 \times 300 \mathrm{~s}$ and $1 \times 1800 \mathrm{~s})$ as dynamic framing. Select subtract as delays. Choose in the advanced settings field 128 as sinogram width, 3 as span, 79 as ring difference and dead time correction.

4. For PET reconstruction, use two-dimensional ordered subset expectation maximization (2D-OSEM) with a generate, apply and save scatter sinogram, 4 iteration and Fourier for rebinning as reconstruction algorithm. Select $128 \times 128$ as matrix size and use 1 as image zoom, all as frames and all as segments.

\section{Procedure of manual blood sampling}

1. Perform manual blood sampling $30 \mathrm{~s}, 60 \mathrm{~s}, 90 \mathrm{~s}, 600 \mathrm{~s}$ and $1800 \mathrm{~s}$ after starting the imaging acquisition.

NOTE: Increasing the number of manual blood draws especially within the first minute after tracer injection is highly recommended if possible. Therefore the blood sample volume has to be reduced to $20-30 \mu \mathrm{L}$ per sample ${ }^{6}$.

1. Open the first three-way valve and collect $100 \mu \mathrm{L}$ of arterial blood into a capillary blood collection EDTA tube $30 \mathrm{~s}$ after tracer injection. Repeat for the other time points. Determine the weight of the empty tube and blood filled tube.

2. Measure the activity (counts/time unit) of the whole blood for $180 \mathrm{~s}$ in a well counter, which is later cross calibrated to obtain data in $\mathrm{kBq} / \mathrm{mL}$. Record the start time of the well counter measurement. Calculate the activity of whole blood for each time point of the manual blood sampling in $\mathrm{kBq} / \mathrm{mL}$, apply decay correction and transfer the data in a time activity curve.

\section{Procedure of the online blood sampling}

1. Place the tube into the detector using the tube guide. Start the blood sampler software (e.g., PSAMPLE) and open the acquisition interface. Ensure that the computer of the online blood sampling setup and of the PET/CT is time synchronized.

2. Press the start button exactly $60 \mathrm{~s}$ before the tracer is injected to acquire enough data for background correction. Save the raw data via the save button in the PMOD database after the measurement.

3. For correction and calibration of the online blood data, switch to the correction interface. Enable the decay correction and select $18 \mathrm{~F}$. Define the start time of image acquisition and enable the average button to perform background correction. Activate the calibration and type in the previously determined calibration factor (see section 7.1 ).

4. Save the corrected and calibrated blood data using the save TAC button and choose the file blood.crv. This file can then be loaded as whole blood input curve into the kinetic modeling tool and kinetic modeling can be performed. Decouple the catheters from the extra corporal shunt system.

5. Detach the animal from the PET/CT scanner and euthanize with pentobarbital.

NOTE: In this experiment, animals were euthanized after the measurements as brains were used for in vitro analyses in the experimental design. With this setup, repeated measurements in longitudinal studies are also implementable ${ }^{7}$. Use a completely new tube system for the next animal.

\section{Image derived Input function}

1. Open the Fuse it tool on PMOD. Load the PET Image as input and the CT as reference. Click already matched.

2. Open the voxel of interest (VOI) tool. Place the cursor within the ascending aorta in the CT. Click predefined spherical VOI. Define a radius of exactly $0.7 \mathrm{~mm}$. Extract the time activity information with the VOI statistic button and copy the averaged values to clipboard.

\section{Procedure of cross calibration of the twilite system, PET/CT and well counter}

1. Twilite-PET/CT-calibration

NOTE: The presented workflow for calibration of the twilite is partly based on the procedures described in the reference manual of the PSAMPLE module of PMOD.

1. Fill a syringe with approximately $100 \mathrm{MBq}$ of $\left[{ }^{18} \mathrm{~F}\right] \mathrm{FDG}$. Measure the exact activity $A_{F}$ with a calibrated dose calibrator and document it together with date and time of the measurement and the volume of the full syringe. The recorded time is the reference time point for all decay corrections to be performed.

2. Fill a beaker with $500 \mathrm{~mL}$ of tap water. The exact volume is determined by the weighing method. Measure the weight $m_{e}$ of the empty beaker with an appropriated and calibrated precision scale (at least accuracy class II). Fill the beaker with the tap water and measure the weight $m_{f}$ of the full beaker.

3. Calculate the volume $V_{b}$ of the beaker by using the difference of the mass and the density of tap water $\left(r=0.998 \mathrm{~g} / \mathrm{mL}\right.$ at $\left.20{ }^{\circ} \mathrm{C}\right)$ :

$V_{b}=\frac{m_{f}-m_{e}}{\rho}$

4. Inject the $\left[{ }^{18} \mathrm{~F}\right] \mathrm{FDG}$ into the filled beaker and refill the empty syringe to its original volume with inactive tap water and measure the activity $A_{E}$ of the refilled syringe in the dose calibrator. The activity concentration $c_{b}$ of the solution in the beaker is given by $c_{b}=\frac{A_{F}-A_{E}}{V_{b}}[\mathrm{kBq} / \mathrm{mL}]$, which should be approximately $200 \mathrm{kBq} / \mathrm{mL}$. 
5. Fill a $50 \mathrm{~mL}$ conical centrifuge tube with the solution from the beaker (avoid large air bubbles) and place it centrally in the field of view of the PET/CT scanner. Fill a catheter identical to the type used in the PET/CT imaging experiment and place it in the tube guide of the twilite system. Fill the catheter with the tracer solution from the beaker using the peristaltic pump.

6. Start the measurement of the time activity curve as described in section 5 , using the same parameter for integration time, and rebinning as in the experiment, without a catheter guide inside the measurement head. This step ensures the acquisition of enough data for appropriate background correction. After $2 \mathrm{~min}$, without stopping the data acquisition of the twilite system, place the catheter guide with the filled tube into the measurement head, and continue the data acquisition for about 5 min.

7. Start a $10 \mathrm{~min}$ PET acquisition of the $50 \mathrm{~mL}$ conical centrifuge tube in parallel followed by a standard CT acquisition for attenuation correction. Reconstruct a static PET image of the $50 \mathrm{~mL}$ conical centrifuge tube using the same PET reconstruction algorithm and parameters described in section 3. Use a post processing imaging tool (e.g., PVIEW) and place a cylindrical VOI covering approximately $70 \%$ of the volume inside of the reconstructed PET images of the $50 \mathrm{~mL}$ conical centrifuge tube. Extract the mean activity concentration $C_{P E T}$ in $\mathrm{kBq} / \mathrm{mL}$ within the VOI.

8. Go back to the blood sampler software and use the calibration mode to correct the acquired TAC for decay, branching fraction and background. Add all the necessary information for nuclide, activity concentration and the PET acquisition start time. Internally, the software extracts the count rate measured with the twilite system $\left(C R_{\text {twilite }}\right)$ and calculates the cross calibration factor for PET and twilite system $\left(C F_{P E T / \text { twilite }}\right)$ :

$C F_{P E T / \text { twilite }}=\frac{c_{P E T}}{C R_{\text {twilite }}}\left[\frac{\mathrm{kBq} / \mathrm{mL}}{\mathrm{cts} / \mathrm{s}}\right]$

NOTE: It is important that the same isotope is used for both calibration and PET/CT experiments, as the branching fraction varies between the different isotopes, which is corrected for in the PET reconstruction process. This procedure has to be repeated regularly in terms of quality control, if important components of the system are changed (e.g., tubes, acquisition and reconstruction parameters) and after repair works.

\section{PET/CT-well counter calibration}

1. To calculate the calibration factor $C F_{\text {well-counter }}$ of the well counter, use the same activity solution that has been produced in the beaker for the calibration of the twilite system. Wait approximately $6 \mathrm{~h}$ to allow reduction of specific activity by decay to minimize dead time effects of the scintillation detector of the well counter. Lid the beaker to avoid evaporation.

2. Calculate the exact time difference to the reference time point and determine the actual activity concentration $c_{b}\left(t_{+}\right)$of the solution of the beaker by decay correcting the original activity concentration. Pipette predefined volumes $\left(V_{\text {sample }}\right)$ that are identical to the volume of the blood samples measured within the experiments (e.g., $200 \mu \mathrm{L}$ ), from the beaker into five safe-lock tubes. Measure the activity of each of the five tubes with the well counter for $180 \mathrm{~s}$

NOTE: If the coefficient of variation for a single measurement is greater than $1 \%$, the measurement time should be increased. Record the measured count rate in counts per minute [cpm] for each tube and the measurement start time. Perform a decay correction.

3. Calculate the calibration factor $C F_{\text {well-counter }}$ for each measurement by dividing the decay corrected count rate $C R_{\text {well-counter }}$ of the well counter by the decay corrected activity concentration of the beaker $c_{\text {beaker }(t+)}$ :

$$
C F_{\text {well-counter }}=\frac{C R_{\text {well-counter }}}{C_{b}\left(t_{+}\right) \cdot V_{\text {sample }}}\left[\frac{C p m}{B q}\right]
$$

4. Average the five calibration factors to obtain the mean calibration factor.

\section{Representative Results}

The setup of the shunt system is displayed in Figure 2. Representative results of the continuous blood sampling data compared to manual blood sampling data in three wildtype rats over a time span of $30 \mathrm{~min}$ are presented in Figure 3A,C. At the beginning of the continuous blood sampling an initial peak (maximum of radioactivity concentration) can be seen at $5 \mathrm{~s}$ after tracer injection. Afterwards, the activity in the blood declines rapidly and reaches a plateau at about $15 \mathrm{~min}$. In the manual blood sampling data the detected Peak is smaller and the plateau is not easily to define (Figure 3A,C). The comparison of the continuous blood sampling to the image-derived data is displayed in Figure 3B,D. In the imagederived data, the peak and the starting point of the plateau are clearly visible, nevertheless the maximum of the peak is smaller compared to continuous blood sampling data for all animals (Figure 3B,D).

A sub-optimal outcome of continuous blood sampling with our setup is shown in Figure 3E,F. At the beginning of the continuous blood sampling, no data acquisition within the first $3.5 \mathrm{~min}$ was possible due to blood clotting. By disconnecting the tube system at connector orange and floating with heparinized saline solution, the flow in the tube system was restarted and the measurement continued. A peak can be seen at about $4 \mathrm{~min}$, which does not record the maximum of radioactivity in blood (Figure 3E,F). Manual blood sampling (Figure 3E) and image-derived analyses (Figure $\mathbf{3 F}$ ) were still possible and comparable to the correct outcomes. 

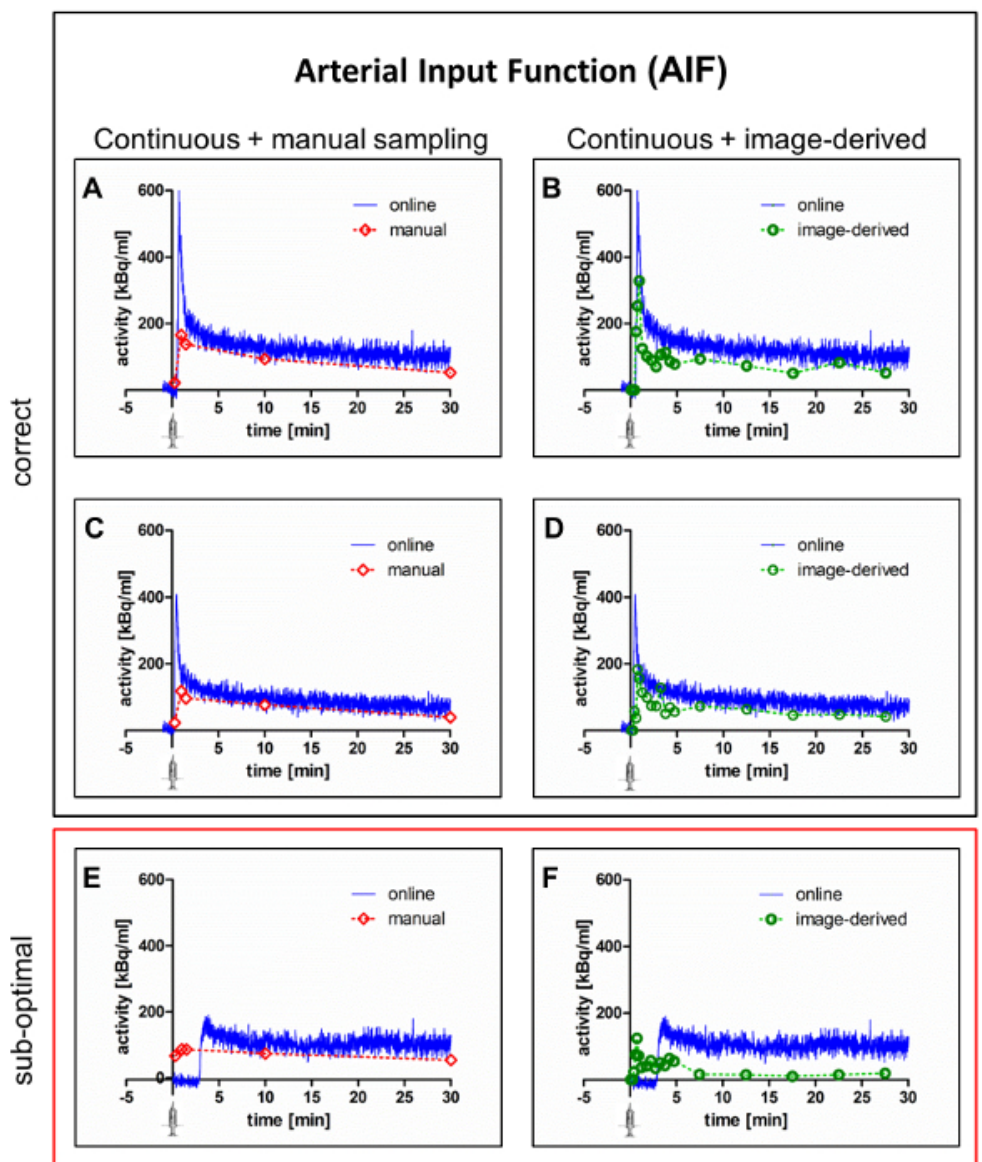

Figure 3: Representative results of continuous blood sampling compared to manual blood sampling. Typical arterial input functions derived from continuous blood sampling compared to manual blood sampling (left column) and continuous blood sampling compared to the image-derived approach (right column) are shown. Panels A-D demonstrate the results of correct implementation of the protocol in two different animals. Panels $\mathbf{E}$ and $\mathbf{F}$ illustrates a sub-optimal outcome of the measurement. All data shown were corrected for the cross-calibration factor and the background. Please click here to view a larger version of this figure.

\section{Discussion}

The presented results are extracted from a larger-scale project on neuronal activity in a transgenic animal model of Huntington's disease compared to wildtype rats. Altogether 30 transgenic and wildtype rats were catheterized and manual and online blood sampling in parallel to $\left[{ }^{18}\right.$ F]FDG-PET/CT was performed. Three AIFs of wildtype rats are shown here to demonstrate the range of possible outcomes of the protocol. The results of the complete project on changes of neuronal activity in an animal model of Huntington's disease will be published elsewhere.

The here described method enables fast and accurate continuous blood sampling in a big cohort and provides a gapless AIF for kinetic modeling of dynamic PET/CT data in small animals. An external blood circulation is generated to detect actual time activity in the blood of the animals; consequently a loss of blood is avoided. The surgical procedure is based on Jespersen et al. ${ }^{8}$ and was modified to meet the needs for arterial blood sampling during the PET/CT measurements. The shunt system was validated by Weber et al. ${ }^{9}$. With the here used setup, an external blood volume of about $1.1 \mathrm{~mL}$ is running through the detector-pump system. A rat aged 4 month has a total blood volume of about $30 \mathrm{~mL}$. The diameter of the femoral vein and artery is approximately $0.45-0.6 \mathrm{~mm}^{10}$ and needs to be a little starched to insert the catheter used.

The AIF can also be measured via sporadic manual blood collection or be reconstructed from early time points of the PET images itself (imagederived). Both approaches were performed with the here presented data and compared to the continuous blood sampling.

In comparison to manual blood sampling, with online blood sampling a noticeable higher temporal resolution (here: 1800 data points per 30 $\mathrm{min}$ ) becomes possible. Manual blood draws (here: 5 data points per $30 \mathrm{~min}$ ) are limited to the blood volume present in the small animal, as these samples are not pumped back into the circulation of the animal. Moreover, a maximum interval of $10-15 \mathrm{~s}$ is technically implementable and important information for kinetic modeling is missed. This can also be seen in the presented data, as a difference in the detected maximum of continuous and manual blood sampling is obvious (Figure 3A,C,E). With online blood sampling the detected peak was higher than with the image-derived input function of the ascending aorta ${ }^{11}$ (Figure 3B,D,F). The Imaged-derived input function is restricted to the spatial resolution of PET scanners which results in partial volume effects ${ }^{12}$ and is affected by the reconstructed time frames.

A general advantage of this continuous blood sampling procedure is that the tracer can be applied via the catheter, which is less prone to disturbance than injection via the lateral tail vein. Keep in mind that the tracer should be applied in a moderate volume to prevent the tracer from remaining in the beginning of the tube system. To ensure that no activity is remaining in the dead volume of the T-piece, it is flushed with 
heparinized saline solution afterwards. Moreover, the usage of an infusion pump is advised as it enables adjustment of the speed of the tracer injection and can contribute to more coordinated acquisition of the maximum radioactivity peak with manual blood sampling ${ }^{13}$.

There are a few possible difficulties that might occur during protocol processing and can be handled by the following troubleshooting. A suboptimal position of the catheters might lead to an incomplete execution of the protocol, therefore ensure that they are accurately fixed with the proximal suture and that the catheter is pushed $2-3 \mathrm{~cm}$ proximal into the vessel. In addition, fibrin adhesive can be used. Also formation of thrombi can clog the catheters. This can be handled by increasing the heparin concentration and subsequent flushing of the catheters or the tube system. Such a sub-optimal outcome due to clogging of the catheters is shown in the results, the maximum peak is missed (Figure 3E). Another critical point concerning animal protection and well-being is the length of the extracorporeal blood flow. It is therefore suggested to reduce the length of the tube system to a minimum.

When blood sampling is performed, three corrections of the resulting AIF have to be taken into account. First, plasma correction. Tracers equilibrate between plasma and blood cells, mainly erythrocytes. Depending on how fast these diffusion processes are, the available tracer is mainly present in plasma. For some tracers, the ratio of plasma to whole blood needs to be considered, such as more lipophilic ones. In these cases, plasma activity has to be determined. If $\left[{ }^{18} \mathrm{~F}\right] \mathrm{FDG}$ is used, there is no need to centrifuge the blood to determine the plasma activity, as it equilibrates very fast between plasma and red blood cells and the availability of $\left[{ }^{18} \mathrm{~F}\right] \mathrm{FDG}$ in plasma is similar to that in the whole blood Secondly, metabolite correction. Many tracers are metabolized in whole blood and some of these metabolites are still radioactively labeled ${ }^{14}$. This fraction is present in the AIF but is not available for tissue uptake. For some tracers metabolites need to be determined in whole blood or plasma and the AIF needs to be corrected. Thirdly, dispersion correction. Dispersion is caused by several factors, including (a) the systematic time difference between the tracer arrival times in the tissue relative to the peripheral sampling site (delay correction) and (b) and the smearing of the shape of the AIF, as the tracer transport within the tube system is influenced by its first order lag (PT 1$)$ kinetics. Several corrections based on deconvolution have been proposed, mainly based on the model by lida et al. ${ }^{15}$, but most of them are susceptible to noise. A correction method which circumvents deconvolution and is therefore less prone to noise has been proposed by Munk et al. ${ }^{16}$. The necessary measurements to estimate the correction parameters have to be performed for every combination of tubing and tracer used. Dispersion correction should be done before time delay correction ${ }^{17}$. However, mainly fast tissue perfusion processes are affected by dispersion and it has also been shown, that for modeling of $\left[{ }^{18} \mathrm{~F}\right] \mathrm{FDG}$ studies a dispersion correction is not absolutely necessary ${ }^{18}$. Therefore, in the presented examples the dispersion correction of the AIF has not been applied.

A proper calibration of the on-site dose calibrator and its regular quality control is a prerequisite for the type of cross calibration procedures presented here. However, if the activity administered to the animal is measured with the same dose calibrator, any deviation in accuracy will be cancelled out, provided that the deviation is constant and the complete cross calibration procedure has been followed, including nuclide-specific corrections (e.g., for varying half-life or different branching ratio). Using such a calibration procedure for harmonizing PET/CT systems used in human health care and research, an accuracy of at least $5-10 \%$ could be achieved $^{19,20}$.

The calibrated and corrected AIFs generated by successful implementation of this protocol enable quantification of PET/CT data for the characterization of animal disease models, testing of new therapy options, establishment of new tracers, and transferring of existing tracers into another species. Seemingly, continuous blood sampling in $\left[{ }^{18}\right]$ FDG-PET/CT in rats delivers the most reliable information for the calculation of the input in bio-kinetic modeling. By taking into account the individual metabolism, especially liver clearance, a more precise assessment of the relevant pathological or therapeutical effects is possible. With this practicable protocol, a higher efficiency of preclinical PET/CT data analysis is easily implementable.

\section{Disclosures}

The authors have nothing to disclose.

\section{Acknowledgments}

The authors gratefully acknowledge Susann Lehmann, Iloana Klamfuß and Petra Wolff for animal housing and care and Matthias Wyss for support during the establishment of the online blood sampling system. The small animal PET/CT was funded by the Deutsche Forschungsgemeinschaft (INST 2268/6-1 FUGG).

\section{References}

1. Schain, M. et al. Arterial input function derived from pairwise correlations between PET-image voxels. Journal of cerebral blood flow and metabolism : official journal of the International Society of Cerebral Blood Flow and Metabolism. 33 (7), 1058-1065, (2013).

2. Schain, M., Zanderigo, F., Mann, J.J., Ogden, R.T. Estimation of the binding potential BPND without a reference region or blood samples for brain PET studies. Neurolmage. 146, 121-131, (2017).

3. Bentourkia, M. Determination of the Input Function at the Entry of the Tissue of Interest and Its Impact on PET Kinetic Modeling Parameters. Molecular Imaging and Biology. 17 (6), 748-756, (2015).

4. Phelps, M.E. PET. Springer New York. New York, NY. (2004).

5. Laforest, R. et al. Measurement of input functions in rodents: challenges and solutions. Nuclear Medicine and Biology. 32 (7), 679-685, (2005).

6. Napieczynska, H. et al. Impact of the Arterial Input Function Recording Method on Kinetic Parameters in Small-Animal PET. Journal of Nuclear Medicine: Official Publication, Society of Nuclear Medicine. 59 (7), 1159-1164, (2018).

7. Sijbesma, J.W.A. et al. Novel Approach to Repeated Arterial Blood Sampling in Small Animal PET: Application in a Test-Retest Study with the Adenosine A1 Receptor Ligand [(11)C]MPDX. Molecular Imaging and Biology: MIB: the Official Publication of the Academy of Molecular Imaging. 18 (5), 715-723, (2016). 
8. Jespersen, B., Knupp, L., Northcott, C.A. Femoral arterial and venous catheterization for blood sampling, drug administration and conscious blood pressure and heart rate measurements. Journal of Visualized Experiments. (59), (2012).

9. Weber, B., Burger, C., Biro, P., Buck, A. A femoral arteriovenous shunt facilitates arterial whole blood sampling in animals. European Journal of Nuclear Medicine and Molecular Imaging. 29 (3), 319-323, (2002).

10. Liu, H.-L. Microvascular anastomosis of submillimeter vessels-a training model in rats. Journal of Hand and Microsurgery. 5 (1), 14-17, (2013).

11. van der Weerdt, A.P. et al. Image-derived input functions for determination of MRGlu in cardiac (18)F-FDG PET scans. Journal of Nuclear Medicine: Official Publication, Society of Nuclear Medicine. 42 (11), 1622-1629, at <http://www.ncbi.nlm.nih.gov/pubmed/11696630> (2001).

12. Alf, M.F. et al. Quantification of brain glucose metabolism by 18F-FDG PET with real-time arterial and image-derived input function in mice. Journal of Nuclear Medicine: Official Publication, Society of Nuclear Medicine. 54 (1), 132-138, (2013).

13. Eriksson, O. et al. A computerized infusion pump for control of tissue tracer concentration during positron emission tomography in vivo pharmacokinetic/pharmacodynamic measurements. BMC Medical Physics. 8, 2, (2008).

14. Burger, C., Buck, A. Tracer kinetic modelling of receptor data with mathematical metabolite correction. European Journal of Nuclear Medicine. 23 (5), 539-545, (1996).

15. lida, $\mathrm{H}$. et al. Error analysis of a quantitative cerebral blood flow measurement using $\mathrm{H} 2(15) \mathrm{O}$ autoradiography and positron emission tomography, with respect to the dispersion of the input function. Journal of Cerebral Blood Flow and Metabolism: Official Journal of the International Society of Cerebral Blood Flow and Metabolism. 6 (5), 536-545, (1986).

16. Munk, O.L., Keiding, S., Bass, L. A method to estimate dispersion in sampling catheters and to calculate dispersion-free blood time-activity curves. Medical Physics. 35 (8), 3471-3481, (2008).

17. Meyer, E. Simultaneous correction for tracer arrival delay and dispersion in CBF measurements by the $\mathrm{H} 215 \mathrm{O}$ autoradiographic method and dynamic PET. Journal of Nuclear Medicine: Official Publication, Society of Nuclear Medicine. 30 (6), 1069-1078, at <http:/l www.ncbi.nlm.nih.gov/pubmed/2786948> (1989).

18. Lanz, B., Poitry-Yamate, C., Gruetter, R. Image-derived input function from the vena cava for 18F-FDG PET studies in rats and mice. Journal of Nuclear Medicine: Official Publication, Society of Nuclear Medicine. 55 (8), 1380-1388, (2014).

19. Geworski, L. et al. Multicenter comparison of calibration and cross calibration of PET scanners. Journal of Nuclear Medicine: Official Publication, Society of Nuclear Medicine. 43 (5), 635-639 (2002).

20. Boellaard, R. Standards for PET image acquisition and quantitative data analysis. Journal of Nuclear Medicine: Official Publication, Society of Nuclear Medicine. 50 Suppl 1, 11S-20S, (2009). 